\title{
Periodic Limb Movements in Sleep is Associated with Increased Mortality
}

\author{
Jae-Won Choi ${ }^{1}$, Ji Soo Song ${ }^{2}$, Yu Jin Lee', and Do-Un Jeong ${ }^{1 凶}$ \\ ${ }^{1}$ Department of Psychiatry and Center for Sleep and Chronobiology, Seoul National University College of Medicine and Hospital, Seoul, \\ Republic of Korea \\ ${ }^{2}$ Department of Biological Basis of Behavior, University of Pennsylvania, PA, USA
}

\begin{abstract}
Objective To elucidate the association between periodic limb movements in sleep (PLMS) and mortality.
Methods Nocturnal polysomnographic recordings of 1,344 subjects obtained from 1995 to 2008 were reviewed. The subjects were divided into four groups based on PLMS and insomnia: reference group (PLMS $\leq 5$ ), insomnia group (PLMS $\leq 5$ with insomnia symptoms), $5<$ PLMS $\leq 15$ group, and the PLMS $>15$ group. We searched each subject's Identification Number in the death records from the Statistics of Korea, the national bureau of statistics, to determine deaths in the cohort that occurred prior to December, 2013. Cox-proportional hazard regression and Kaplan-Meier survival curve analyses were used to compare mortality among the four groups.

Results Hazard ratios (HRs) in the $5<$ PLMS $\leq 15$ and PLMS $>15$ groups were significantly higher than that in the reference group before adjusting for age and gender [HR, 3.37; 95\% confidence interval (CI), 1.73-6.55; $\mathrm{p}<0.001$; HR, 5.77; 95\% CI, 3.24-10.29; $\mathrm{p}<0.001$ ]. Only the PLMS $>15$ group had a higher mortality rate than that in the reference group after adjusting for age, gender, and sleep efficiency (HR, 1.99; 95\% CI, 1.06-2.21; $\mathrm{p}=0.033$ ).

Conclusion These results suggest that PLMS may be associated with increased mortality. Psychiatry Investig 2017;14(5):669-673

Key Words Periodic limb movements in sleep, Mortality.
\end{abstract}

\section{INTRODUCTION}

Periodic limb movements in sleep (PLMS) are a sleep-related phenomenon involving repetitive stereotypical flexion and extension movements of the lower extremities. ${ }^{1}$ PLMS was originally called nocturnal myoclonus, but this term is no longer used. According to American Academy of Sleep Medicine criteria, PLMS are diagnosed if a series of at least four movements with amplitude $\geq 8 \mathrm{uV}$ occurs in a row, lasting $0.5-10 \mathrm{~s}$ in duration and recurs every 5-90 s. The Periodic Limb Movement Index (PLMI), which assesses the frequency of PLMS, is the number of PLMSs per hour of total sleep time.

Received: August 25, 2016 Revised: October 18, 2016

Accepted: November 19, 2016 Available online: July 26, 2017

$\triangle$ Correspondence: Do-Un Jeong, $\mathrm{MD}, \mathrm{PhD}$

Department of Psychiatry and Center for Sleep and Chronobiology, Seoul National University College of Medicine, 101 Daehak-ro, Jongno-gu, Seoul 03080, Republic of Korea

Tel: +82-2-2072-2456, Fax: +82-2-744-7241, E-mail: jeongdu@snu.ac.kr

(a) This is an Open Access article distributed under the terms of the Creative Commons Attribution Non-Commercial License (http://creativecommons.org/licenses/bync/4.0) which permits unrestricted non-commercial use, distribution, and reproduction in any medium, provided the original work is properly cited.
More than five PLMI per hour is considered abnormal according to the International Classification of Sleep Disorders first edition (ICSD-1). However, this cutoff value is not based on any data, and is ineffective to separate symptomatic from asymptomatic PLMS due to the high prevalence of asymptomatic PLMS. Thus, PLMI $>15 / h$ in adults and $>5 / h$ in children is a requisite diagnostic criterion for periodic limb movement disorder (PLMD) in the ICSD-3. A diagnosis of PLMD requires polysomnographic (PSG) results, clinical sleep disturbance, and exclusion of other sleep disorders.

The prevalence of PLMI $>15$ is $5-8 \%$ in adults and increases with age. ${ }^{2-4}$ PLMS is commonly observed in the elderly in the absence of other sleep disturbances. ${ }^{4,5}$ PLMS is detected in $>80 \%$ of patients with restless leg syndrome (RLS) on PSG. ${ }^{6}$ However, PLMS that occur in subjects without RLS, are found in $25 \%$ of individuals undergoing routine nocturnal PSG.? Although PLMS and RLS can occur independently, their frequent association suggests that they share a common etiology. ${ }^{8.9}$ Despite the close relationship between RLS and PLMS, previous studies reported that even after adjustment of RLS symptoms and sleep disturbance, the increased frequency of 
PLMS itself is significantly associated with systolic heart failure or end-stage renal disease and could independently increase mortality. ${ }^{10,11}$ Therefore, our study focused on the direct relationship between PLMS and mortality, and excluded occurrence of RLS or insomnia symptoms as variables. Furthermore, previous studies that investigated the relationship between PLMS and mortality had small sample sizes (30-218 subjects) and relatively short observational periods (mean duration, 32.9-48 months). ${ }^{10-12}$ No study has conducted a long-term investigation of mortality based on PLMS severity in a population with a high incidence of PLMS and in a relatively large sample. The objective of this study was to elucidate the association between PLMS and mortality in patients with PLMS based on nocturnal PSG, in relatively large sample size and long observational period.

\section{METHODS}

\section{Subjects}

We retrospectively reviewed the medical records and PSG recordings of 4,517 subjects who visited the Center for Sleep and Chronobiology at Seoul National University Hospital from April 1995 to December 2008 who were $\geq 15$-years-old and underwent full-night PSG. Subjects with obstructive sleep apnea [Apnea-Hypopnea Index (AHI) $\geq 5$ ], RBD, or narcolepsy were excluded from the study. The following criteria were used to determine the insomnia group: difficulty initiating or maintaining sleep for at least 3 months; certified psychiatrists' confirmation of insomnia symptoms based on the Diagnostic and Statistical Manual of Mental Disorders, fourth edition criteria; maintenance of a $\mathrm{AHI}<5$ and $\mathrm{PLMI} \leq 5$; and no sign of any other sleep disorder, such as narcolepsy or RBD based on PSG. Among the patients excluded from the insomnia group, subjects with $\mathrm{AHI}<5, \mathrm{PLMI} \leq 5$, and no symptoms of any other sleep disorder, were included in the reference group. These subjects were referred for complaints of snoring, sleep disturbances or abnormal behaviors during sleep, among other, but were normal according to PSG. We classified the subjects into two groups based on the PLMI: PLM group $1(5<\mathrm{PLMI} \leq 15)$, which did not meet the ICSD-3 but did meet the ICSD-2 criteria for PLMD and PLM group 2 (PLMI $>15$ ). Subsequently, we investigated the death of these subjects that occurred up to December 31, 2013. We obtained approval of the Seoul National University Institutional Review Board.

\section{Nocturnal polysomnography}

All subjects underwent PSG at a sleep laboratory. Sleep stage, respiratory events, and movement events were scored by trained sleep technicians and sleep physicians according to standard criteria. Apnea was defined as a complete or near- complete ( $\geq 90 \%)$ cessation of airflow $\geq 10$ s. Hypopnea was defined as a $>30 \%$ reduction in the respiratory signal lasting at least $10 \mathrm{~s}$ and associated with oxygen saturation $\geq 3 \%$ from the baseline or an arousal. PLMS were defined as at least four consecutive movements at $25 \%$ of the amplitude of resting legs, lasting $0.5-5 \mathrm{~s}$, and separated by $5-90 \mathrm{~s}$ apart. Sleep efficiency (SE), sleep latency (SL), and wake after sleep onset time (WASO) were calculated.

\section{Mortality data}

We searched for each subject's Korean Identification Number and name in the death records from the Statistics of Korea, the national bureau of statistics, to determine deaths in the cohort that occurred prior to December 31, 2013. Date of death and primary cause of death were noted in these records.

\section{Statistical analysis}

The subjects' characteristics were compared with the PLMI and presence of insomnia using the $\chi^{2}$ test for categorical variables and analysis of variance for continuous variables and the Bonferroni test for post-hoc comparisons. Cox proportional hazard regression analysis was employed to assess the associations between PLMI and insomnia and mortality risk; results were estimated as hazard ratios (HRs) with $95 \%$ confidence intervals (CIs). Unadjusted models and models adjusted for age, gender, and SE were evaluated. A Kaplan-Meier survival analysis was conducted to compare survival rates among the groups. SPSS ver. 21.0 software (SPSS Inc., Chicago, IL, USA) was used for the analysis. A p-value $<0.05$ was considered significant.

\section{RESULTS}

Of the 4,517 subjects, 2,924 had AHI $\geq 5,119$ had RBD, and 130 had narcolepsy and were excluded. Finally, the study included 1,344 subjects (mean age, 47.0 years; $51.1 \%$ male). Among the 1,344 subjects, 149 (11\%) were classified into the PLM group 1 (5<PLMI $\leq 15), 217$ (16\%) were placed in PLM group 2 (PLMI> $15)$, and 564 (42\%) were put in the insomnia group. The insomnia group and PLM group 2 (PLMI>15) showed significantly lower SE (\%) and higher WASO ( $\mathrm{min}$ ) than those of the reference group. The insomnia group had significantly longer SL than that in the reference group and PLM group 1 ( $5<$ PLMI $\leq 15$ ). All subjects were followed for a mean of 11.8 years to assess their mortality, from the date of PSG until death or study termination. Of the 1,344 subjects studied, 110 died during the follow-up period (Table 1). Cause of death was cancer in $35(31.8 \%)$ subjects, trauma in 19 (17.3\%), cardiovascular disease in seven (6.4\%), and other causes in 49 (44.5\%) subjects.

The HR of all-cause mortality was significantly elevated in 
Table 1. General characteristics of the PLM, insomnia and reference groups

\begin{tabular}{|c|c|c|c|c|c|c|c|}
\hline & $\begin{array}{l}\text { Reference } \\
(\mathrm{PLMI} \leq 5)\end{array}$ & Insomnia & $\begin{array}{l}\text { PLM group } 1 \\
(5<\mathrm{PLMI} \leq 15)\end{array}$ & $\begin{array}{c}\text { PLM group } 2 \\
(\mathrm{PLMI}>15)\end{array}$ & Total & $\mathrm{p}$-value & Bonferroni \\
\hline $\mathrm{N}$ & 414 & 564 & 149 & 217 & 1,344 & & \\
\hline Age & $39.1 \pm 14.4$ & $48.0 \pm 12.2$ & $51.7 \pm 12.6$ & $56.4 \pm 13.3$ & $47.0 \pm 14.4$ & $<0.001$ & Ref. $<$ Ins. $<1<2$ \\
\hline Gender (male, \%) & $272(65.7)$ & $247(43.8)$ & $70(47.0)$ & $98(45.2)$ & $687(51.1)$ & $<0.001$ & Ref. >Ins., 1,2 \\
\hline PLMI & $0.5 \pm 1.1$ & $0.5 \pm 1.0$ & $9.3 \pm 2.9$ & $44.7 \pm 37.0$ & $8.6 \pm 21.9$ & $<0.001$ & Ref., Ins. $<1<2$ \\
\hline Death (\%) & $16(3.9)$ & $34(6.0)$ & $19(12.8)$ & $41(18.9)$ & $110(8.2)$ & $<0.001$ & Ref., Ins. $<1,2$ \\
\hline Duration (days) & $4,313.4 \pm 1,542.3$ & $4,561.3 \pm 1,442.3$ & $4,211.6 \pm 1,582.4$ & $3,707.9 \pm 1,651.5$ & $4,308.4 \pm 1,550.6$ & $<0.001$ & Ref., Ins., $1>2$ \\
\hline SE (\%) & $81.6 \pm 18.6$ & $76.2 \pm 16.6$ & $77.5 \pm 16.7$ & $75.5 \pm 18.0$ & $77.9 \pm 17.6$ & $<0.001$ & Ref. $>$ Ins., Ref. $>2$ \\
\hline $\mathrm{SL}(\min )$ & $25.6 \pm 46.4$ & $42.3 \pm 57.8$ & $28.6 \pm 40.4$ & $34.1 \pm 46.3$ & $34.3 \pm 51.4$ & $<0.001$ & Ref. $<$ Ins., Ins. $>1$ \\
\hline WASO (min) & $\begin{array}{c}70.5 \pm 79.7 \\
(281)\end{array}$ & $\begin{array}{c}93.3 \pm 69.0 \\
(434)\end{array}$ & $\begin{array}{c}90.8 \pm 69.6 \\
(106)\end{array}$ & $\begin{array}{c}95.1 \pm 72.0 \\
(133)\end{array}$ & $\begin{array}{c}86.5 \pm 73.4 \\
(954)\end{array}$ & $<0.001$ & Ref. $<$ Ins., Ref. $<2$ \\
\hline
\end{tabular}

PLM: periodic limb movement, PLMI: periodic limb movement index, SE: sleep efficiency, SL: sleep latency, WASO: wakefulness after sleep onset time, Ref.: reference, Ins.: insomnia

Table 2. Hazard ratios for all-cause mortality in each group

\begin{tabular}{|c|c|c|c|c|c|c|}
\hline \multirow{2}{*}{ Groups } & \multicolumn{2}{|l|}{ Model 1} & \multicolumn{2}{|l|}{ Model 2} & \multicolumn{2}{|l|}{ Model 3} \\
\hline & Hazard ratio $(95 \% \mathrm{CI})$ & p-value & Hazard ratio $(95 \% \mathrm{CI})$ & $\mathrm{p}$-value & Hazard ratio $(95 \% \mathrm{CI})$ & $\mathrm{p}$-value \\
\hline Reference $($ PLMI $\leq 5)$ & Reference & & Reference & & Reference & \\
\hline Insomnia & $1.45(0.80-2.63)$ & 0.218 & $0.99(0.54-1.80)$ & 0.965 & $0.89(0.48-1.64)$ & 0.704 \\
\hline PLM group $1(5<\mathrm{PLMI} \leq 15)$ & $3.37(1.73-6.55)$ & $<0.001$ & $1.90(0.96-3.73)$ & 0.064 & $1.77(0.90-3.50)$ & 0.098 \\
\hline PLM group 2 (15<PLMI) & $5.77(3.24-10.29)$ & $<0.001$ & $2.10(1.12-3.92)$ & 0.020 & $1.99(1.06-2.21)$ & 0.033 \\
\hline
\end{tabular}

Model 1: before adjustments, Model 2: after adjusting for age and gender, Model 3: after adjusting for age, gender, and sleep efficiency. PLM: periodic limb movement, PLMI: periodic limb movement index, CI: confidence interval

PLM group $1(5<$ PLMI $\leq 15)$ and PLM group 2 (PLMI $>15)$. However, only PLM group 2 (PLMI $>15$ ) had a significantly increased HR (HR, 2.10; 95\% CI, 1.12-3.92; $\mathrm{p}=0.020$ ) after adjusting for age and sex. This statistical significance remained after adjusting for SE (Table 2). The Kaplan-Meier survival curves are presented in Figure 1. The survival rates of PLM group $1(5<\mathrm{PLMI} \leq 15)$ and PLM group 2 (PLMI $>15)$ were significantly lower than those in the insomnia and reference groups $(\mathrm{p}<0.001)$; in particular, the survival rate of PLM group 2 (PLMI $>15)$ was significantly lower than that of PLM group 1 $(5<\mathrm{PLMI} \leq 15)(\mathrm{p}=0.048)$. No difference in survival was found between the insomnia and reference groups $(\mathrm{p}=0.474)$. Mean survival times $(95 \% \mathrm{CI})$ for the reference group, insomnia group, and PLM groups 1 and 2 were 18.1 years (17.8-18.7), 18.0 years $(17.7-18.2), 17.0$ years $(16.2-17.7)$, and 15.8 years (15.0-16.6), respectively. The Cox regression analysis indicated that male sex was not associated with increased all-cause mortality (HR, 1.453; 95\% CI, 0.99-2.13; $\mathrm{p}=0.055$ ), but a high PLMI was associated with increased all-cause mortality (HR, 1.009; 95\% CI, 1.00-1.01; $\mathrm{p}<0.001$ ) (Table 3).

\section{DISCUSSION}

A higher PLMI score was associated with increased allcause mortality in this single center-based population who underwent PSG. Subjects with $5<$ PLMI $\leq 15$ and PLMI $>15$ experienced respective 3.4- and 5.8-fold increases in all-cause mortality compared with that of subjects with no sleep disorder. However, this association remained significant only in subjects with PLMI $>15$ after adjusting for age, gender, and SE, so an increased PLMI was associated with a 2.1-fold increase in all-cause mortality among subjects with PLMI $>15$. No significant association was detected between insomnia and all-cause mortality.

This is the first study showing an association between the PLMI and mortality regardless of the presence of medical disease. Previous studies showed that PLMS is associated with mortality but was limited to patients with heart and renal diseases. ${ }^{10-12}$ In addition, the subjects were only divided into two groups, such as PLMI $<5$ and PLMI $\geq 5$. Unlike previous studies, our study subjects were divided into three groups, and those with $5<\mathrm{PLMI} \leq 15$ were not associated with increased mortality after adjusting for age, gender, and SE.

Although the pathophysiology of PLMS is not well defined, a few studies have demonstrated that PLMS increases the risk for developing hypertension, heart diseases, renal disease, and stroke. ${ }^{10,11,13,14}$ One of the hypotheses for the pathophysiology of PLMS is a dopamine deficit. ${ }^{15}$ Hypofunction of the dopaminergic pathway may lead to increased sympathetic activity and a subsequently increased risk of developing hy- 


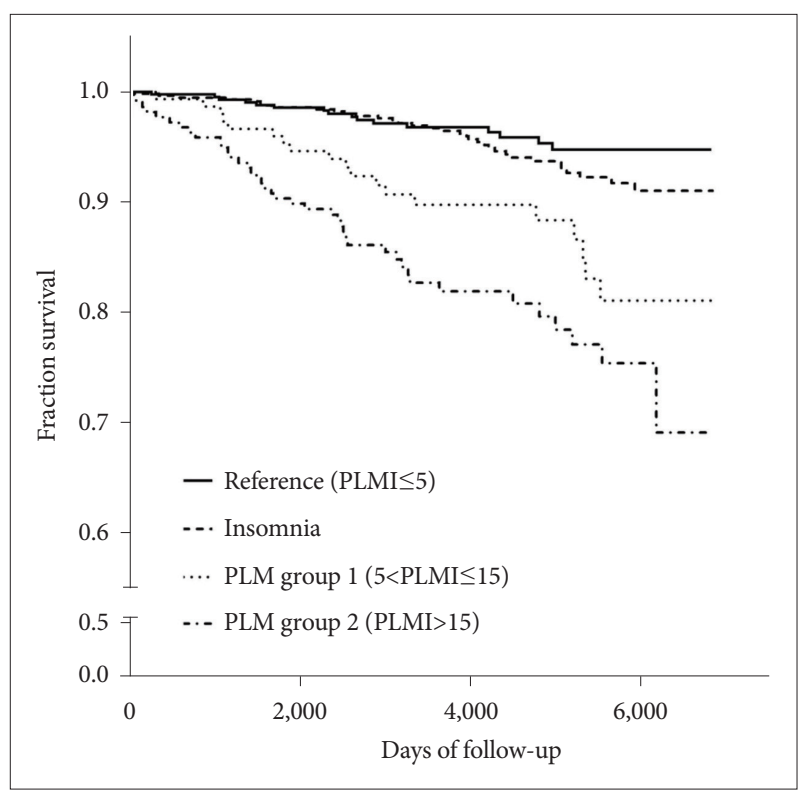

Figure 1. Kaplan-Meier survival curves for all-cause mortality. Kaplan-Meier estimated survival rate for all-cause mortality according to the PLMI and insomnia. Survival rate was significantly lower in group $2(P L M l>15)$ than in all other groups $(p<0.001)$. Group $1(5<P L M I \leq 15)$ had lower survival rates than those in the insomnia and reference groups $(p=0.001$, both). The survival rate did not differ between the insomnia and reference groups $(p=0.474)$. PLMI: periodic limb movement index.

Table 3. Hazard ratios of related variables in the entire study population $(\mathrm{N}=1,344)$

\begin{tabular}{lcr}
\hline \multicolumn{1}{c}{ Variables } & Hazard ratio $(95 \% \mathrm{CI})$ & p-value \\
\hline Age & $1.076(1.06-1.09)$ & $<0.001$ \\
Gender (male) & $1.453(0.99-2.13)$ & 0.055 \\
PLMI & $1.009(1.00-1.01)$ & $<0.001$ \\
\hline PLMI: periodic limb movement index, CI: confidence interval
\end{tabular}

pertension, heart disease, and stroke. ${ }^{14}$ PLMS accompanied by arousals are associated with transient rises in heart rate and blood pressure that cause nocturnal hypertension and nondipping blood pressure. ${ }^{16}$ Nondipping blood pressure has been associated with increased cardiovascular morbidity and mortality. ${ }^{17}$ Thus, PLMS and arousals may be associated with increased mortality. However, we only experienced seven (6.4\%) cardiovascular-cause deaths, and the most common cause of death was cancer (31.8\%). Since most common cause of death in this study was cancer, we conducted Cox proportional hazard regression analysis between PLM group 2 (PLMI $>15$ ) and reference group, but there are no significant results after adjusting for age, sex and SE (HR, 1.16; 95\% CI, 0.45-3.02; p= 0.775). All-cause mortality was significantly high among PLM group 2 (PLMI $>15$ ). Possible explanation is involved in the increased sympathetic activity associated with PLM based on previous report on the relation of hypertension with renal cell carcinoma. ${ }^{18}$ In addition, some studies showed that hy- pertension could be a risk factor for colorectal, prostate, brain cancer, and malignant melanoma. ${ }^{19}$

Our results do not establish a causal relationship between increased PLMS and increased mortality. It is possible that PLMS is simply associated with disturbed sleep architecture. Nevertheless, increased PLMS remained a significant risk factor for mortality after adjusting for SE, suggesting that PLMS may have contributed to increased mortality regardless of disturbed sleep architecture. Subjects who do not complain of difficulty initiating or maintaining sleep but have PLMI $>15$, in other words do not meet the diagnostic criteria for PLMD, should be considered for treatment to reduce PLMS.

Another interesting finding was that only PLMI $>15$ was associated with increased mortality, which is a new PLMS level in the ICSD-3 defining an increase. This means that the ICSD3 criteria on PLMS frequency may reflect the adverse effects of PLMS on individual health better than prior versions of the criteria.

A limitation of this study is that we collected data from clinical samples instead of the general population, which could have led to a selection bias. Because our statistical analyses were based on retrospective review of medical records and PSG results, information on confounding factors including medical and psychiatric disorders, ordinary sleep habits and hypnotics use were limited. Also, despite the close relationship between RLS and PLM and the fact that diagnosis of PLMD requires assessment of insomnia or daytime sleepiness symptoms, not being able to adjust for such variables is a critical limitation of this study. Therefore, an extensive study that compiles statistics on such confounding variables will further clarify the association between increased PLMS and increased mortality. Another limitation of this study is that its retrospective nature precluded recruiting healthy participants for the control group. Despite these limitations, the large sample size and the long follow-up period add to the validity of our results.

In conclusion, the results suggest that increased PLMS is associated with an increased risk of all-cause mortality independently of SE and SL. Although our results do not provide a causal relationship between increased PLMS and increased mortality, further research is needed to determine the pathophysiology, clinical progress, and therapeutic effects of PLMS for decreased mortality.

\section{REFERENCES}

1. Hornyak M, Feige B, Riemann D, Voderholzer U. Periodic leg movements in sleep and periodic limb movement disorder: prevalence, clinical significance and treatment. Sleep Med Rev 2006;10:169-177.

2. Scofield H, Roth T, Drake C. Periodic limb movements during sleep: population prevalence, clinical correlates, and racial differences. Sleep 2008;31:1221-1227.

3. Bliwise DL, Carskadon MA, Dement WC. Nightly variation of period- 
ic leg movements in sleep in middle aged and elderly individuals. Arch Gerontol Geriatr 1988;7:273-279.

4. Ancoli-Israel S, Kripke DF, Klauber MR, Mason WJ, Fell R, Kaplan O. Periodic limb movements in sleep in community-dwelling elderly. Sleep 1991;14:496-500.

5. Carrier J, Frenette S, Montplaisir J, Paquet J, Drapeau C, Morettini J. Effects of periodic leg movements during sleep in middle-aged subjects without sleep complaints. Mov Disord 2005;20:1127-1132.

6. Allen RP, Picchietti DL, Garcia-Borreguero D, Ondo WG, Walters AS, Winkelman JW, et al. Restless legs syndrome/Willis-Ekbom disease diagnostic criteria: updated International Restless Legs Syndrome Study Group (IRLSSG) consensus criteria--history, rationale, description, and significance. Sleep Med 2014;15:860-873.

7. Chervin RD, Hedger KM. Clinical prediction of periodic leg movements during sleep in children. Sleep Med 2001;2:501-510.

8. Stefansson H, Rye DB, Hicks A. A genetic risk factor for periodic limb movements in sleep. N Engl J Med 2007;357:639-647.

9. Winkelmann J, Schormair B, Lichtner P. Genome-wide association study of restless legs syndrome indentifies common variants in three genomic regions. Nat Genet 2007;39;1000-1006.

10. Yumino D, Wang H, Floras JS, Newton GE, Mak S, Ruttanaumpawan $\mathrm{P}$, et al. Relation of periodic leg movements during sleep and mortality in patients with systolic heart failure. Am J Cardiol 2011;107:447-451.

11. Benz RL, Pressman MR, Hovick ET, Peterson DD. Potential novel predictors of mortality in end-stage renal disease patients with sleep disorders. Am J Kidney Dis 2000;35:1052-1060.
12. Jung HH, Lee JH, Baek HJ, Kim SJ, Lee JJ. Nocturnal hypoxemia and periodic limb movement predict mortality in patients on maintenance hemodialysis. Clin J Am Soc Nephrol 2010;5:1607-1613.

13. Espinar-Sierra J, Vela-Bueno A, Luque-Otero M. Periodic leg movements in sleep in essential hypertension. Psychiatry Clin Neurosci 1997;51:103-107.

14. Walters AS, Rye DB. Review of the relationship of restless legs syndrome and periodic limb movements in sleep to hypertension, heart disease, and stroke. Sleep 2009;32:589-597.

15. Vetrugno R, D’Angelo R, Montagna P. Periodic limb movements in sleep and periodic limb movement disorder. Neurol Sci 2007;28(Suppl 1): S9-S14.

16. Sieminski M, Partinen M. A relationship between periodic limb movements in sleep and high nocturnal blood pressure values in patients with insomnia. J Clin Sleep Med 2016;12:865-869.

17. Ohkubo T, Hozawa A, Yamaguchi J, Kikuya M, Ohmori K, Michimata $\mathrm{M}$, et al. Prognostic significance of the nocturnal decline in blood pressure in individuals with and without high 24-h blood pressure: the Ohasama study. J Hypertens 2002;20:2183-2189.

18. Kocher NJ, Rjepaj C, Robyak H, Lehman E, Raman JD. Hypertension is the primary component of metabolic syndrome associated with pathologic features of kidney cancer. World J Urol 2016;35:67-72.

19. Radisauskas R, Kuzmickiene I, Milinaviciene E, Everatt R. Hypertension, serum lipids and cancer risk: a review of epidemiological evidence. Medicina (Kaunas) 2016;52:89-98. 